\title{
AN INVERSE DYNAMIC METHOD
YIELDING FLEXIBLE P MANIPULATOR STATE TRAJECTORIES
}

\author{
"onz.Soc Kwon and Wayne J. Book \\ George W Wcudruff Scizool of Mechenical En \\ Ge rgia Institute of Techrology \\ Atlanta, GA 30332
}

\begin{abstract}
An inverse dynamic equation for a fl:xible manipulator is derived in a state form. By dividing the inverse system into the causal part and the antic usa. part, we can calculate inrque in the time domain for a cerain end point trajectory, as well as trajectories of all state variables. The open loop control of the inverse dynamic method shows an excellent re:ult in simulation. For practical applications, a control strategy adapting feedback tracking control to the inver: dynamic feedforward control is illustrated, and its good experimental result is presented.
\end{abstract}

\section{Introduction}

Flexible manipulators have been widely discussed in the literat.ure recently. Most of these papers have mentioned the flexible manipulator's advantages such as low mass to payload ratio, use of small actuators, and high travelling speed. However, the inherent flexibility of the manipulatol makes its actual industrial application difficult. In spite of its flexibility, a flexible manipulator should have the capability to follow a given end point trajectory for practical applications. This paper proposes a simple inverse dynamic method which can make the end point of a flexible manipulator accurately follow a given trajectory without overshoot or residual vibration.

The feedback control regulating method, which adds damping effect to the flexible structure is one of typical methods used to suppress the structural vibration of the manipulator in literature. By using joint and strain feedback control, Hastings and Book [7] demonstrated good results in regulating the vibration. Even though the feedback control is able to dampen the residual vibration during settling time, their experiment showed the undershoot, the overshoot, and the flexible vibration in case of a step response. For a step input command, these vibrating phenomena are inevitable with the feedback control scheme because the feedback control signal contains high frequency components, which excite the system natural frequencies. Instead of a step response, a smooth nominal trajectory can be used as the joint reference command of tracking control. However, the strain feedback tracking control requires the desired trajectory values of flexible mode variables which match the joint trajectory value. Because of lacking flexible mode values, assigning zero values to the desired values of flexible mode states has been acceptable for the nominal trajectory tracking control to suppress the vibration during motion. In other words, reference commands are given to the flexible manipulator to follow the trajectcry like a rigid manipulator. Even though the increase $d$ damping by the feedback control regulates the vibration, such unrealistic commands cannot avoid the generation of the vibration.

To avoid the above faedback control problems, De Luca and Siciliano [6] suggested a joint based inversion control scheme. This method showed good tracking result for a certain joint trajectory. However, it couldn't be extended for an end point trajectory following control because of nonminimum phase system characteristics. Oosting and Dickerson [8] demonstrated a calculation method of the torque to follow a smooth trajectory for a simple lumped parameter two link flexible manipulator.

To make the end point of a flexible manipulator follow a given trajectory, Bayo [2] proposed a new approach. For a given end point acceleration profile, the required torque was calculated by solving the inverse dynamic equation in the frequency domain through the inverse fast Fourier transform. The inverse dynamic system considering the end point acceleration as input and the joint torque as output is a noncausal system since the output (torque) must begin before the input (end point acceleration) begins. Therefore, this inverse dynamic method provides a noncausal solution torque which acts before the tip moves, and after the tip stops. In spite of excellent results, his method has a drawback because it requires heavy computation for the transformation of a dynamic model and an input trajectory from the time domain to the frequency domain and the inverse transformation of the output to the time domain. To reduce such computation burden, Bayo and Moulin [4] recently introduced the convolution integral method to solve the inverse dynamic equation.

Asada and $\mathrm{Ma}$ [1] derived an inverse dynamic equation by using assumed mode functions for a general $n$-link case. As the transfer function of a flexible manipulator between the input torque and the output end point position is a non-minimum phase system, it has some positive real value zeros. These zeros become positive poles of the inverse dynamic system transfer function. They cause the inverse system to be unstable 
if the inverse system output is restricted to causal solutions. In the reference [1], they used rigid body torque to show nonlinear effect without solving the inverse dynamic equation.

In this paper, we introduce a new simple inverse dynamic method, which relieves the calculation burden considerably. With this method, we can calculate the required torque in the time domain. In addition to that, we can generate very natural trajectories of flexible mode coordinates, which match the given end point trajectory dynamically. These trajectories can be and are used as reference commands of flexible coordinates for feedback tracking control.

In following sections, we first describe a direct dynamic model of a single link manipulator by using assumed mode methods. And we derive an inverse dynamic system equation from the dynamic model in a state space form. By dividing the inverse system into the causal part and the anticausal part, we can integrate the inverse dynamic differential equation of each part separately in the time domain. Next, this inverse dynamic method is implemented on the single link flexible manipulator in Fig. 1 through simulations. Its results are compared with the output of other typical control methods. Finally, the controller design with the inverse dynamic method is presented, and its experimental results are discussed.

\section{Modeling}

A single link flexible manipulator having planar motions is described as shown in Fig. 1. The rotating inertia of the servo motor, the tachometer, and the clamping hub are modeled as the hub inertia lh. The payload is modeled as the end mass Me. Even though flexible beam damping exists, it is ignored in modeling.

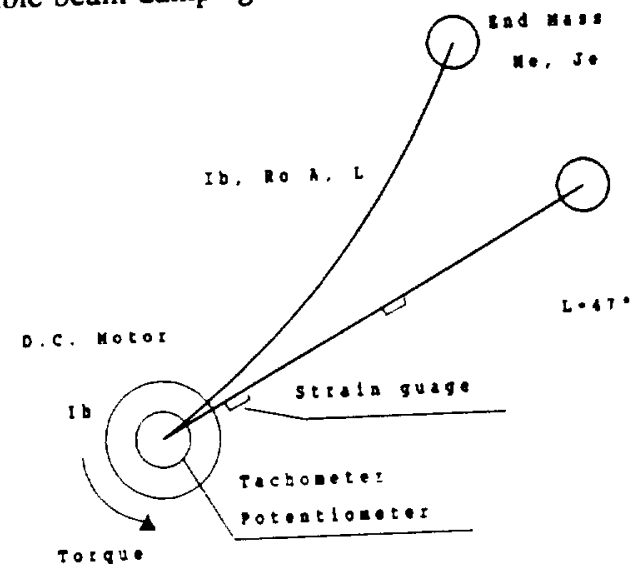

Fig 1. A single link flexible manipulator

To derive equations of motion of the manipulator, we describe the position of a point on the beam with a combination of a virtual rigid body motion and flexible deflections using a Bernoulli-Euler beam model. The virtual rigid body motion is represented by the motion of the moving coordinate which is attached to the beam. The flexible deflections are described by finite series of assumed modes with respect to that moving reference frame. Defining the rigid body motion is important because different mode shape functions have to be used according to the choice of the rigid body coordinate. Several authors [7,9] used the rigid body coordinate which is attached at the base hub like a) of Fig. 2, and used the clamp-free boundary condition mode functions. Other authors [5] defined the rigid body mode coordinate to pass through the center of mass of the beam, and used pin-free mode functions. And others [1] let the rigid body coordinate pass through the end point, and used the pin-pin mode functions. All of these definitions for the rigid body mode can be valid because appropriate mode functions, which satisfy the geometric boundary conditions can be chosen for each case.

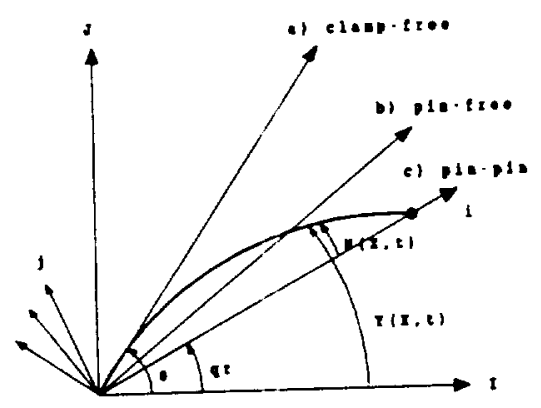

Fig 2. The kinematic descriptions of flexible manipulator coordinates

In this paper, the rigid body mode coordinate which passes through the end point of the beam is selected, and the pin-pin mode functions are used to describe deflections. In order to obtain a accurate model with a small number of modes, we have considered more accurate boundary conditions such as the joint hub inertia and the end mass when we derive the mode shape functions. For the inverse dynamic model, two mode functions of pin, hub inertia-pin,end mass boundary conditions are used. The reason why this rigid body coordinate is selected is that the end point position of the beam can be expressed by the rigid body mode variable, and this simple representation of the end point position makes the derivation of the inverse dynamics equation easy.

The velocity of any point of the beam can be expressed as follows.

$$
\begin{aligned}
& r=x \dot{x}+w j \quad \text { where } w=w(x, t) \\
& r=\left\{x \dot{q}_{r}+\dot{w}\right] j-w \dot{q}_{r} i
\end{aligned}
$$




$$
\text { Then } \begin{aligned}
\dot{r} \dot{r} & =\left(x \dot{q}_{r}+\dot{w}\right)^{2}+w^{2} \dot{q}_{r}^{2} \\
& -\left(x \dot{q}_{r}+\dot{w}\right)^{2} \quad \text { where } w<x
\end{aligned}
$$

The kinetic energy of a beam is obtaisad oy the integration the valocity product term. Its s scond term generates centrifuga' and coriolis forces in $t$ le $d \cdot$ narr ic equation. In this single link case, the econd

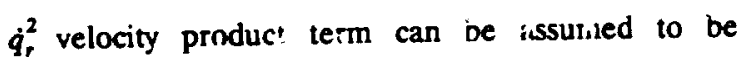
negligible to provide a linearized model except in the case of very high rotational speed.

As we can consider $x$ as $\phi_{0}(x)$ and $q_{f}(t)$ as $q_{0}(t)$, let's introduce another variable $y$ which represents the total displacement of the point on the beam as shown in Fig. 2.

$$
\begin{aligned}
& y=x q_{r}+w-x q_{r}(t)+\sum_{i=1}^{n} \phi_{i}(x) q_{i}(t) \\
& -\sum_{i=0}^{n} \phi_{i}(x) q_{i}(t) \\
& \text { Then } \left.\quad \dot{r} \dot{r}-y^{2}-\mid \sum_{i=0}^{n} \phi_{i} \dot{q}_{i}\right\}^{2}
\end{aligned}
$$

Thus the kinetic energy and the elastic potential energy will written as

$$
\begin{aligned}
T & =\frac{1}{2} \int_{0}^{l} j(x, t)^{2} d x+\frac{1}{2} I_{h} \dot{y}^{\prime}(x-0, t)^{2} \\
& +\frac{1}{2} M e y^{j}(x-l, t)^{2}+\frac{1}{2} J e \dot{y}^{\prime}(x-l, t)^{2} \\
& =\frac{1}{2} \int_{0}^{1} \sum_{i=0}^{n}\left(\phi, \dot{q}_{i}\right)^{2} d x+\frac{1}{2} l_{h}\left(\sum_{i=0}^{n} \phi_{i}^{\prime}(0) \dot{q}_{i}\right)^{2} \\
& +\frac{1}{2} M e\left(\sum_{i=0}^{n} \phi_{i}(l) \dot{q}\right)^{2}+\frac{1}{2} J e\left(\sum_{i=0}^{n} \phi_{i}^{\prime}(l) \dot{q}_{i}\right)^{2} \\
K & -\frac{1}{2} E I \int_{0}^{l} w^{\prime \prime}(x, l)^{2} d x-\frac{1}{2} E I \int_{0}^{l} y^{\prime \prime}(x, t)^{2} d x \\
& -\frac{1}{2} E I \int_{0}^{l}\left(\sum_{i=0}^{n} \phi_{i}^{\prime \prime} q_{i}\right)^{2} d x
\end{aligned}
$$

And the virtual work is expressed as

$$
\begin{aligned}
\delta W & =\tau \cdot \delta y^{\prime}(x-0, t)=\tau\left(\sum_{i=0}^{n} \Phi_{i}(0) \delta q_{i}\right. \\
& =\sum_{i=0}^{n} Q_{i} \delta q_{i}
\end{aligned}
$$

Therefore, the generalized force $Q_{i}$ is expressed as follows:

$$
Q_{i}-\phi(0) \cdot \tau
$$

By using Lagrange s equations of motion, the dynamic equation of a flexible maripulator is obtained with generalized coordinates.

$$
\begin{gathered}
\frac{d}{d}\left(\frac{\partial T}{\partial \dot{q}_{i}}\right)-\frac{\partial T}{\partial q_{i}}+\frac{\partial V}{\partial q_{i}}-Q_{i}, \quad i-G, 1, \ldots, n \\
{[M] \ddot{q}+[K]_{q}-[B] \tau}
\end{gathered}
$$

$$
\begin{aligned}
& \text { where } \\
& {[M]=\left[\begin{array}{cc}
M_{i j} & \ldots \\
\vdots & \ddots
\end{array}\right], \quad[K]-\left[\begin{array}{ccc}
0 & 0 & \ldots \\
0 & K_{i j} & \ldots \\
\vdots & \vdots & \ddots
\end{array}\right]} \\
& {[B]-\left[\begin{array}{cc}
B_{i} \\
\vdots
\end{array}\right], \quad q-\left\{\begin{array}{l}
q_{i} \\
\vdots
\end{array}\right\}, \quad i-0,1, \ldots n} \\
& M_{i j}=\left(\begin{array}{l}
\rho A \int_{0}^{\prime} \phi_{i}(x) \phi_{j}(x) d x \\
+I_{h} \phi_{i}^{\prime}(0) \phi_{j}^{\prime}(0) \\
+M_{i} \phi_{i}(D) \phi_{j}(l) \\
+J_{e} \phi_{i}^{\prime}(D) \phi_{j}^{\prime}(l)
\end{array}\right) \\
& K_{i}=E I \int_{0}^{\prime} \phi_{i}^{\prime \prime}(x) \phi_{j}^{\prime \prime}(x) d x, \\
& i j-0, . . n
\end{aligned}
$$

The mass and stiffness matrices are represented in these general forms which are valid for different definitions of rigid body modes. For the viscous friction of a joint, the damping term is included. The dynamic equation can be divided into a rigid body motion part and a flexible motion part as follows:

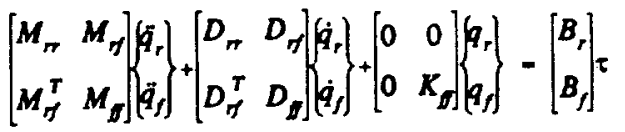

$$
\begin{aligned}
& \text { where } q_{r}-q_{0} \text {; rigid body coord., } \\
& q_{f}=\left\{\begin{array}{l}
q_{1} \\
\vdots
\end{array}\right\} ; \text { flexible mode coord }
\end{aligned}
$$

For a state space form, we obtain the following dynamic equation. 


$$
\begin{aligned}
& \dot{X}=\left[\begin{array}{cc}
0 & I \\
M^{-1} K & M^{-1} D
\end{array}\right] X+\left[\begin{array}{c}
0 \\
M^{-1} B
\end{array}\right] \tau \\
& \begin{aligned}
Y & =[C] x+[F] \tau \\
\text { where } \quad X & =\left\{q_{p}, q_{,}, \dot{q}_{,}, \dot{q}_{f}\right\}^{T} \\
& =\left\{q_{0}, q_{1}, \cdots, \dot{q}_{0}, \dot{q}_{1}, \cdots\right\}^{T}
\end{aligned}
\end{aligned}
$$

From now on, we call this dynamic equation the direct dynamic equation to distinguish it from the inverse dynamic equation which is derived at the next section.

\section{Inverse dynamic equations}

Base on the above the direct dynamic equations, we will derive the inverse dynamic equation. Eqn. (1) can be written in two parts.

$$
\begin{aligned}
& {\left[M_{r}\right] \bar{q}_{r}+\left[M_{r}\right] \ddot{q}_{f}+\left[D_{r}\right] \dot{q}_{r}+\left[D_{r}\right] \dot{q}_{f}-\left[B_{r}\right] \tau } \\
& {\left[M_{r}\right]^{T} \ddot{q}_{r}+\left[M_{f j}\right] \ddot{q}_{f}+\left[D_{r j}\right]^{T} \dot{q}_{r}+\left[D_{f j}\right] \dot{q}_{f}+\left[K_{f}\right] q_{f} } \\
= & {\left[B_{f}\right] \tau }
\end{aligned}
$$

From Eqn. (3), torque is give by

$$
\tau-[B]^{-1}\left[\left[M_{n}\right] \vec{q}_{r}+\left[M_{n} d \vec{q}_{f}+\left[D_{r}\right] \dot{q}_{r}+\left[D_{n}\right] \dot{q}_{f}\right]\right.
$$

Substitution of above Eqn. (3a) to Eqn. (4) gives the following relations between the flexible coordinates $\mathbf{q}_{\mathbf{r}}$ and the rigid body coordinate $\mathbf{q}_{\mathbf{r}}$

$$
\left[M_{i}\right] \ddot{q}_{f}+\left[D_{i}\right] \dot{q}_{f}+\left[K_{j}\right] q_{f}-\left[B_{i l}\right] \dot{q}_{f}+\left[B_{i 2}\right] \ddot{q}_{r}
$$

$$
\begin{aligned}
& \text { where }\left[M_{i}\right]-\left\{\left[M_{f}\right]-\left[B_{f}\right]\left[B_{r}\right]^{-1}\left[M_{r} d\right\}\right. \\
& {\left[D_{j}\right]-\left\{\left[D_{p}\right]-\left[B d\left[B_{r}\right]^{-1}\left[D_{r} d\right]\right.\right.} \\
& {\left[\boldsymbol{K}_{i}\right]-\left[\boldsymbol{K}_{j}\right]} \\
& {\left[B_{i l}\right]-\left\{\left[B_{f}\right]\left[B_{r}\right]^{-1}\left[D_{n}\right]-\left[D_{r}\right]^{T}\right\}} \\
& {\left[B_{i 2}\right]-\left\{\left[B_{f}\right]\left[B_{r}\right]^{-1}\left[M_{\pi}\right]-\left[M_{r}\right]^{T}\right\}}
\end{aligned}
$$

From Eqn. (4), the acceleration of flexible coordinates are expressed as follows:

$$
\begin{aligned}
\ddot{q}_{f}= & -\left[M_{f f}\right]^{-1}\left[M_{f f}\right]^{T} \bar{q}_{f}-\left[M_{f}\right]^{-1}\left[D_{f f}\right]^{T} \dot{q}_{f} \\
& -\left[M_{f f}\right]^{-1}\left[D_{f f}\right] \dot{q}_{f}-\left[M_{f}\right]^{-1}\left[K_{f}\right] q_{f}+\left[M_{f j}\right]^{-1}\left[B_{f}\right] \tau
\end{aligned}
$$

Substitute this Eqn. (6) to Eqn. (3), then we will get the following Eqn.

$$
\tau=\left[C_{u}\right] q_{f}+\left[C_{b}\right] \dot{q}_{f}+\left[F_{u}\right] \dot{q}_{r}+\left[F_{i 2}\right] \dot{q}_{r}
$$

$$
\begin{aligned}
& \text { where }[G]-\left\{\left[B_{r}\right]-\left[M_{r} d\left[M_{\rho}\right]^{-1}\left[B_{\rho}\right]\right\}^{-1}\right. \\
& {\left[C_{i, 1}\right]-[G]\left\{-\left[M_{\eta j}\right]\left[M_{f}\right]^{-1}\left[K_{f}\right]\right\}} \\
& {\left[C_{i 2}\right]-[G]\left[\left[D_{n}\right]-\left[M_{n} d\left[M_{f f}\right]^{-1}\left[D_{p D}\right]\right\}\right.} \\
& {\left[F_{u}\right]-[G]\left\{\left[D_{n}\right]-\left[M_{\gamma}\right]\left[M_{\rho}\right]^{-1}\left[D_{r}\right]^{T}\right\}} \\
& {\left[F_{[2}\right]-[G]\left[\left[M_{m}\right]-\left[M_{r}\right]\left[M_{d}\right]^{-1}\left[M_{r} d^{\top}\right]\right.}
\end{aligned}
$$

If Eqn. (5) and Eqn. (7) are represented in a state space form, the inverse dynamic equations will be

$$
\begin{aligned}
& \text { Let } \quad x_{i}-\left[q_{\rho} \dot{q}_{j}\right]^{T}, \quad q_{\sigma}-\left[q_{r} \bar{q}_{r}\right\} \\
& \dot{X}_{i}-\left[\begin{array}{cc}
0 & I \\
M_{i}^{-1} K_{i} & M_{i}^{-1} D_{i}
\end{array}\right] X_{i}+\left[\begin{array}{cc}
0 & 0 \\
M_{i}^{-1} B_{u} & M_{i}^{-1} B_{i 2}
\end{array}\right] a_{i r} \\
& =-\left[C_{11}, C_{02}\right] X_{1}+\left[F_{U}, F_{i 2}\right] q_{v} \\
& \dot{X}_{i}-\left[A_{j}\right] X_{i}+\left[B_{i}\right] q_{i} \\
& \tau-\left[C_{j}\right] X_{i}+\left[F_{i}\right] q_{\text {. }}
\end{aligned}
$$

The inverse dynamic equation is obtained in this simple form. By integrating this 1st order differential equation, the required torque can be calculated for the desired endpoint acceleration, and velocity, which are expressed with the rigid body coordinate $q_{r}$ Even though the inverse equation is derived in the simple state space form, the inverse system matrix $A_{i}$ has positive poles (which came from the positive zeros of the transfer function of the direct dynamic system) as well as negative poles. It seems to be impossible to integrate that equation. However, if we relax the solution range of that equation to include noncausal solutions, we can obtain a unique stable solution.

To analyze the inverse system of a flexible manipulator, first of all, let's define several terms: a causal system is the system of which output (impulse response) always occurs after a input (impulse) is given. An anticausal system, on the contrary, always has the output (backwards impulse response) before a input is given (impulse). A noncausal system is defined as the synthesized system of a causal system and an anticausal system.

In order to grasp the meanings of the above definitions, we need to understand the physical phenomena during the motion of a flexible manipulator. If a certain torque profile is applied to the manipulator, there is a unique motion of the end point. On the other hand, if this unique motion of the end point is given as a desired motion, we should be able to get that previous 
- torque profile by using the inverse dynamic equatiors. In some cases, to make the end point follow a certain trajectory profile, we have to preshape (prabend) the flexible manipulator. Therefore, the requi ed iorque, which is necessay to preshape it, has to be ipplied before the end point starts to move. This nea is that the inverse system has anticausal cha acteristics.

- Furthernore, wh:n the flexible maripulator : tops sonie torque should be applied to release the fexible deflection after the es $d p$ int stops. Thi: me'ins t at the inverse system his a causal system characteristics, ton.

_ Thus, such inverse systein can be called a nouzausal system, which is composed of a causal systein and anticausal systen. With this intuitive motivation, we divide the inverse dynamic system into the causal part

- and the anticausal part by using a similarity transformation as follows.

[T]:Orthogonal transformation matrix

$$
\begin{aligned}
& X_{i}-[T] P_{t} \\
& \text { - }\left[T_{c}, T_{\alpha \propto}\right]\left\{P_{c}, P_{\alpha \alpha}\right\}^{T} \\
& {[T]^{-1} A_{i}[T]=\left[\begin{array}{cc}
A_{k} & 0 \\
0 & A_{\text {iec }}
\end{array}\right]} \\
& \left\{\begin{array}{l}
\dot{P}_{c} \\
\dot{P}_{\alpha c}
\end{array}\right\}-\left[\begin{array}{cc}
A_{k c} & 0 \\
0 & A_{\text {ioc }}
\end{array}\right]\left\{\begin{array}{l}
P_{c} \\
P_{\alpha c}
\end{array}\right\}+\left[\begin{array}{l}
B_{i c} \\
B_{i \alpha c}
\end{array}\right] g_{r} \\
& \left\{\begin{array}{l}
\tau_{c} \\
\tau_{\alpha}
\end{array}\right\}-\left[\begin{array}{l}
C_{k} \\
C_{i \alpha c}
\end{array}\right]\left\{\begin{array}{l}
P_{c} \\
P_{\alpha}
\end{array}\right\}+\left[\begin{array}{l}
F / 2 \\
F / 2
\end{array}\right] q_{z} \\
& \tau-\tau_{c}+\tau_{\alpha}
\end{aligned}
$$

Such a coordinate change decouples the inverse system into two subsystems of Eqn. (9). The new variable $P_{\text {represents the coordinate of the causal }}$

- system, and the $P_{\text {ac }}$ does that of the anticausal system. In Eqn. (9), [F] matrix doesn't have to be divided equally. However, it has been done to make the causal system and the anticausal system symmetric. If both

- systems are symmetric and the acceleration profile is skew symmetric, we don't have to integrate both parts. As the output of one system is the same as the reflected output of the other, the total output torque can be obtained by adding one output itself and its reflection.

In general cases, both systems should be integrated separately. For a given end point trajectory

- $q_{r}$ the causal part torque is obtained by integrating the causal part inverse dynamic equations forward from the initial time of the trajectory. However, the anticausal system equations must be integrated backwards from the - final time of the trajectory. The interpretation of this separate integration method to a convolution integral gives good explanations about the backward integration.
The total torque, which is the output of these equations 's obtained by adding the both part $t$ orques as shown in Fig. 5.

As additional outputs, we cou'd build the reference trajects ry of the who'e flexible mode coordinates of the direct dynamic s.'stem from a rigid body mode trajectory. As we can expect in Eqn. (9), the spac: of the full state vector of the direct dynamic system can be divided into thrne si bspaces: the rigid body coordinate subspace, the causal part flexible coordinate subspace, and the anticuusal part flexible coordinate subspace. These subspaces are linearly independent and orthogonal to another. The relations of these spaces are illustrated in Fig. 3, and described by the following Eqn. (10) when two flexible modes are used.

$$
\begin{aligned}
& X=\left[\begin{array}{ll}
1 & 0 \\
0 & 0 \\
0 & 0 \\
0 & 1 \\
0 & 0 \\
0 & 0
\end{array} a_{r}+\left[\begin{array}{llll}
0 & 0 & 0 & 0 \\
1 & 0 & 0 & 0 \\
0 & 1 & 0 & 0 \\
0 & 0 & 0 & 0 \\
0 & 0 & 1 & 0 \\
0 & 0 & 0 & 1
\end{array}\right] X_{1}\right. \\
& X=H_{r} q_{r}+H_{\gamma}\left(T P_{P}\right) \\
& X=H_{r} q_{r}+H_{r} T_{c} P_{c}+H T_{\alpha} P_{\alpha}
\end{aligned}
$$

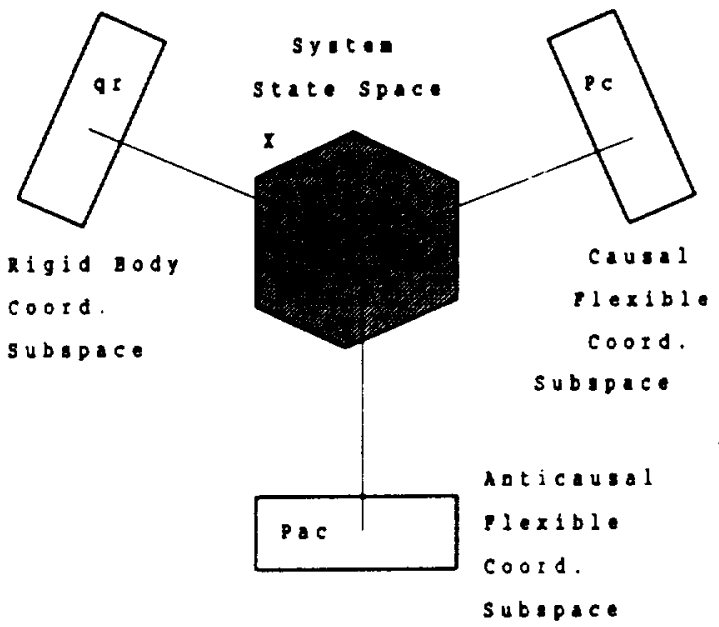

Fig 3. Dimensional analysis of state variables of flexible manipulator dynamic equations

From the end point trajectory, the rigid body coordinate trajectory $q_{r}$ is given, and the flexible coordinate trajectories of $P_{c}$ and $P_{a c}$ are calculated from the integration of Eqn. (g). Thus, the trajectories of the whole states $X$ can be obtained by using Eqn. (10). These trajectory values can be used as reference commands for feedback tracking control. 


\section{Trajectory Generation}

Among the desired trajectories of the manipulator end point, the acceleration, and velocity profile are used as input of the inverse dynamic equations. Theoretically, the inverse dynamic equation can give a torque profile for an arbitrary acceleration profile. However, as Bayo mentioned in the ref. [3], it is important to apply the acceleration profile which doesn't produce high frequency content of the torque. In this paper, several constraints are applied for a desired acceleration profile to get fast motion and no residual vibration. First, the acceleration profile should not have too much high frequency component. If the acceleration changes sharply, the calculated torque profile will contain high peak impulses. This impulse component of the torque may excite natural frequencies of the flexible manipulator. Moreover, it can saturate the actuator, or it may be beyond the actuator bandwidth. Second, the maximum acceleration limit should be chosen not to saturate the actuator. Third, traveling time should be minimum by using the full capacity of the actuator. This third constraint makes the acceleration profile close to a bang bang type which will result in unwanted high frequency problems. Therefore, we have to select the acceleration profile by compromising the profile smoothness and the use the full capacity of the actuator.

Considering the above constraints, the acceleration profile of Fig. 4 was adopted. It is composed of four 3 rd-order polynomial parts and two constant acceleration parts.
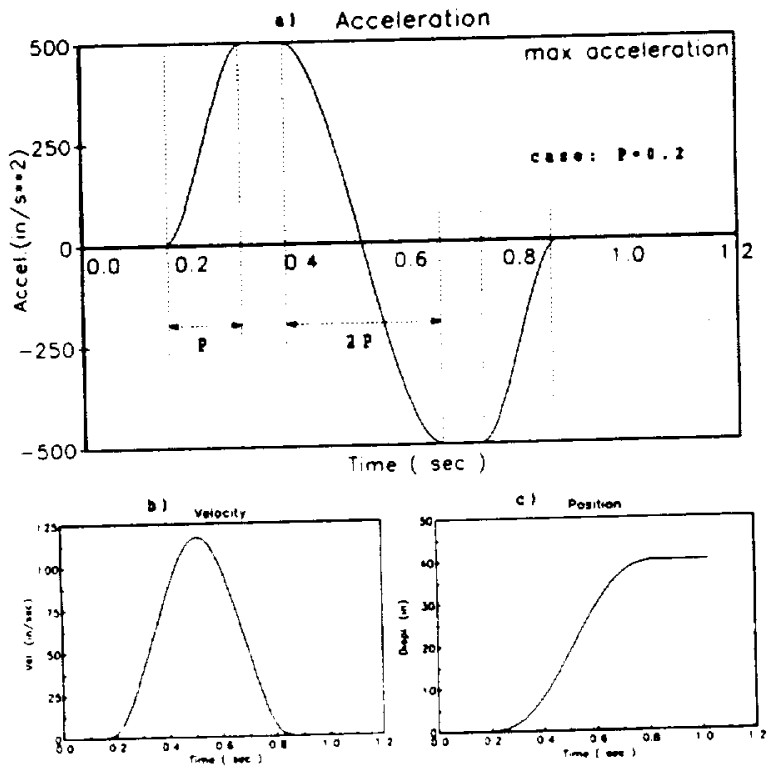

Fig 4. Desired End Point Trajectory

a)Acceleration, b) Velocity, c)Position

The above first and third constraints can be compromised by adjusting the parameter $p$. If $p=0$, the profile will be the bang bang type. And, if $p=0.25$, the profile will be very smooth by being connected with 4 polynomials. In simulation and experiment, the case $p=0.2$ is used.

5. Simulation of the inverse dynamic open loop control and alternative control methods

In this section, we present simulation results to illustrate the performance of the inverse dynamic method. First of all, the total torque profile is calculated from the causal part torque and the anticausal part torque for a given end point acceleration profile as shown in Fig. 5. And the desired trajectories of the output such as joint angle, joint velocity and strains are generated as in Fig. 6.

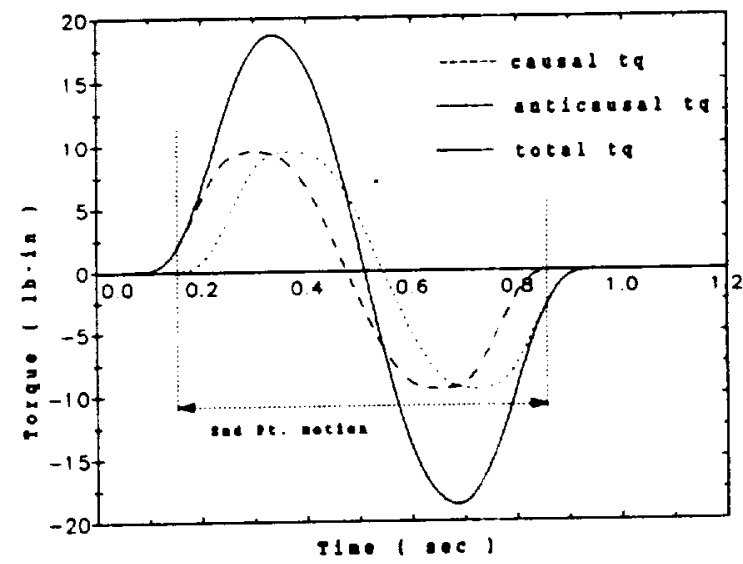

Fig 5. Calculation of torque with the inverse dynamic method

(a)

(b)

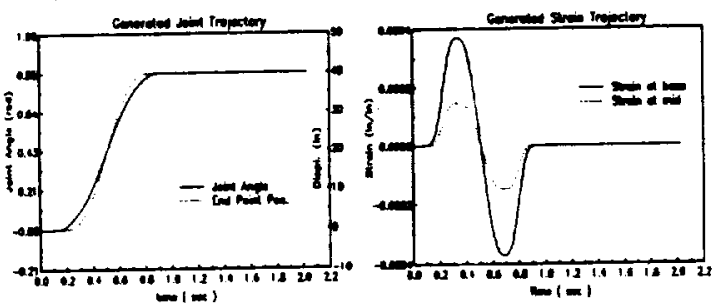

Fig 6. Trajectory generation by using the inverse dynamic method: a) Joint angle, b) Strain at base, and midpoint

The calculated torque was applied to the ideal flexible manipulator model from which the inverse dynamic model was derived. As the results are shown in Fig. 7, the end point follows the desired trajectory exactly, and no undershoot, no overshoot, nor noticeable residual vibration occurs. However, the strain shows oscillations after following the trajectory. It seems to be due to numerical integration errors. 
that a simple joint feedback PD controller performs excellent tracking if it is combined with the inverse dynamic feedforward control, and if the natural trajectory of a joint angle is provided.
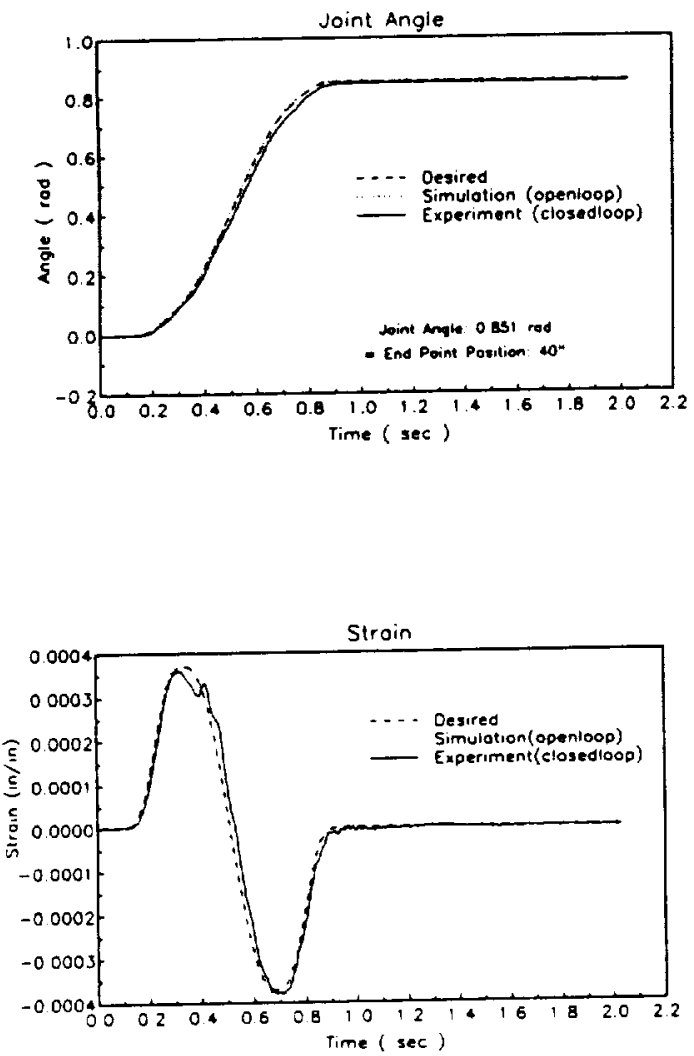

Fig 10. Experimental results of the combined control of the inverse dynamic feedforward control and the joint tracking feedback control: a) Joint angle, b) Strain at base

\section{Conclusion}

The proposed inverse dynamic method provides the simple way to generate the required torque profile in the time domain. Due to the simple computation method, the implementation to the interactive control became easy. Generation of the natural flexible coordinate irajectories is quite useful for feedback control. And the characteristics of flexible coordinates were newly interpreted with causal and anticausal concepts. The feedforward control using the inverse dynamic method shows good tracking performance with a simple joint feedback controller. In other words, this inverse method can relieve the use of many sensors, or feedback calculation burden such as observer reconstruction.

Although several advantages of this method are mentioned, this inverse dynamic method is limited to linear systems. In order to be applied to a multi-link flexible manipulator, this method should be combined with a nonlinear compensation technique.

\section{Acknowledgement}

The Authors would like to acknowledge the support of this research by the National Aeronautics and Space Administration under the Grant No. NAG 1-623.

\section{References}

1. Asada, H. and Ma, Z., Inverse Dynamics of Flexible Robots", Proc. of American Control Conference, pp.23522359, 1989.

2. Bayo, E., "A Finite Element Approach to Control the End-Point Motion of a Single-Link Flexible Robot", Joumal of Robotic Systems, Vol.4, No.1, pp.63-75, 1987.

3. Bayo,E. and Paden, B., "On Trajectory generation for Flexible Robots", Joumal of Robotic Systems, Vol.4, No.2, pp.229-235, 1987.

4. Bayo, E. and Moulin, H., "An Efficient Computation of the Inverse Dynamics of Flexible Manipulators in the Time Domain", Proc. of IEEE Conf. on Robotics and Automation, pp.710-715, 1989.

5. Cannon, R. and Schmitz, E., "Initial Experiments on End Point Control of a Flexible One-Link Robot", Intemational Joumal of Robotics Research, Vol.3, No.3, pp.49-54, 1984.

6. De Luca, A and Siciliano, B., "Joint-Based Control of a Nonlinear Model of a Flexible Arm", Proc of American Control Conference, pp.935-940, Atlanta, June 1988.

7. Hastings, G. and Book, W. J., "Experiments in the Optimal Control of A Flexible Manipulator", Proc of American Control Conference, pp.728-729, Boston, 1985.

8. Oosting, $K$ and Dickerson, S. L., "Simulation of a High-Speed Lightweight Arm", Proc. of IEEE Int. Conf. on Robotics and Automation, pp.494-496, Philadelphia, 1988.

9. Wang, D. and Vidyasagar, M., "Modelling and Control of Flexible Beam Using the Stable Factorization Approach", Proc. of ASME Winter Annual Meeting, Robotics: Theory and Application, Dec., 1986 

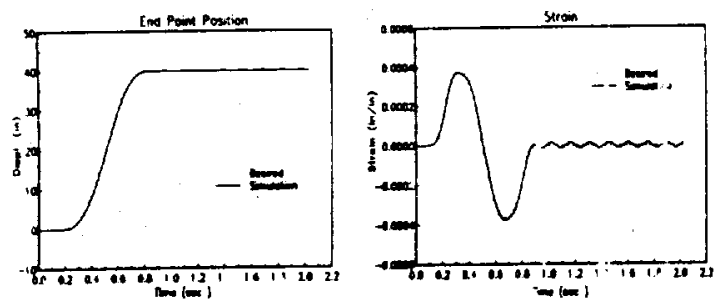

Fig 7. Simulution of the open loop contro wit the inverse dynarnic method

To demonitrate the excellence of this method, we compare the result with those of other typical me:hods. In Fig. 8 (curve i), the result of a collocated joirt PD controller fo: a step input command is den:onstrated. As we can expect, the feedback of position error generates very high peak torque at the beginuing, and it excites the system natural frequencies. Hence, it requires relatively long settling time. It also shows the undershoot and the overshoot of the end point position.

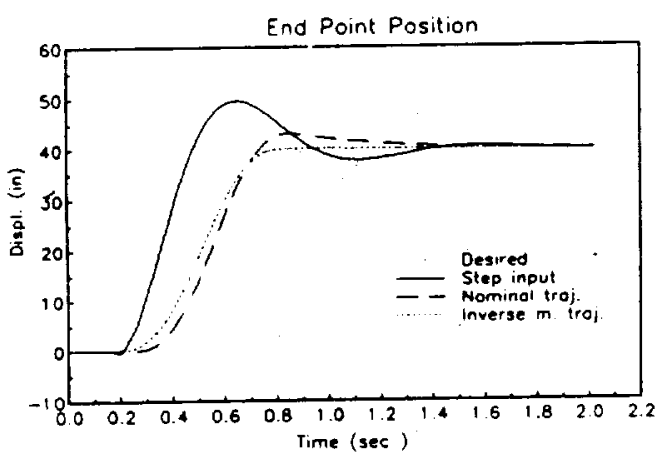

Fig 8. Comparison of typical control methods: a) Joint feedback control with a step input b) Full state feedback tracking control with nominal trajectory c) Full state feedback tracking with the inverse dynamic method trajectory

As a typical alternative method, a nominal joint trajectory tracking control can be used to improve the response instead of a step input. This tracking control is demonstrated with the very smooth nominal position trajectory which is the same as the end point position trajectory. Desired flexible coordinate values were set

to zero, $q_{f}-0, \dot{q}_{f}-0$. The feedback gain was selected by $L Q$ method, and the full state feedback was used. Even though the response is better than that of step input joint feedback case, it still has overshoot and requires settling time. This poor tracking response is due to the unnatural commands which assigns zero values to the flexible coordinate commands. If we give the natural flexible mode trajectcries. which was rbtained from the inverse dynamic method. we can anticipate better response. As it is shown in Fig. 8 (curve $c$ ), by assigning the ratur 1 flexible mode t:ajectory, good trasking performance was achieved.

\section{Experiment}

Although the, open loop control with the inverse dynaraic method showe $d$ the good rerults with an ideal model, it produced large positioning errors with the real system, which has riscous and coulounb friction at the jnint. Therefore, a joint feedback control loop was added to give robustness to the feedionward control of the inverse dynamic method. A friction compensation also was added to cancel the friction effect as shown in Fig. 9.

The above combined control scheme of the inverse dynamic feedforward control and the feedback control was implemented to the experimental single link flexible manipulator, which has 47 inch long arm and a $0.1 \mathrm{lb}$ end mass. It was driven by an Inland D.C. servo motor with a current amplifier. For a fast real time control, A Micro Vax II was used with 12 bit A/D and $D / A$ boards. The off line calculation of a trajectory and a torque profile was also performed in the Micro Vax interactively.

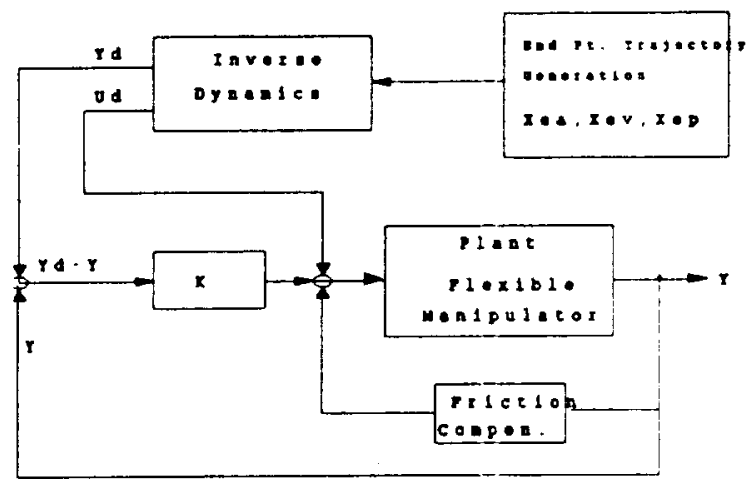

Fig 9. The control scheme of the experiment

By applying the precalculated torque and compensate the friction, and using the feedback of the tracking error at the joint, the excellent result of Fig. 10 was obtained. The flexible manipulator could stop without any overshoot or any residual vibration after it moved 40 inches (48.76 degrees) within less than 1 second. In the strain signal, there exists rough jerk which could be vanished by using more smooth acceleration profile.

Unfortunately, as the end point position sensor was not attached to the system, the end point position couldn't be measured directly. However, the end point tracking performance can be estimated by joint angle tracking result, and the strain on the beam base tracking result. In experiment, only joint angle, and joint velocity signals were used for feedback. The experimental results show 